\title{
EFEITOS DE UM PROGRAMA DE EXERCÍCIOS NA POSTURA VERTEBRAL DE INDIVÍDUOS COM LOMBALGIA ${ }^{1}$.
}

\section{Marcelo Costa de Paula}

Instituto Federal de Educação, Ciência e Tecnologia de Goiás, Luziânia, Goiás, Brasil

Mário Hebling Campos

Universidade Federal de Goiás, Goiânia, Goiás, Brasil

\section{Alexandre Alves Meirelles}

Universidade Estadual de Campinas, Campinas, São Paulo, Brasil

\section{Marco Antonio Alves de Moraes}

Universidade Estadual de Campinas, Campinas, São Paulo, Brasil

\section{René Brenzikofer}

Universidade Estadual de Campinas, Campinas, São Paulo, Brasil

Resumo: O objetivo do presente trabalho foi analisar os efeitos de um programa de exercícios na postura vertebral de dois indivíduos com lombalgia crônica inespecífica (29 e 38 anos; 1.80 e $1.86 \mathrm{~m} ; 86$ e $84 \mathrm{~kg}$ ). A curvatura geométrica $2 \mathrm{D}$ da coluna, no plano sagital, foi quantificada com videogrametria na postura ortostática, na marcha $(1.2 ; 1.5$ e $1.8 \mathrm{~m} / \mathrm{s})$ e na corrida $(2.2 ; 2.6$ e $3.0 \mathrm{~m} / \mathrm{s})$. Após o programa de exercícios, o padrão de movimento da coluna mudou. A amplitude de movimento diminuiu na região lombar. Ocorreu um aumento da lordose lombar e da cifose torácica. Além dessas alterações biomecânicas, houve diminuição do quadro álgico e melhora na função motora, sugerindo a adoção de um padrão de movimento mais apropriado.

Palavras-chave: Locomoção. Biomecânica. Coluna Vertebral. Dor Lombar.

\section{Introdução}

A lombalgia é um distúrbio músculo-esquelético comum na sociedade moderna e tem acarretado graves conseqüências no âmbito da saúde e sócio-econômico (PANJABI, 2003). Cerca de 70 a $85 \%$ da população mundial teve ou terá, em algum momento da vida, um episódio de dor lombar (ANDERSSON, 1999).

Programas de exercícios para aumentar a flexibilidade corporal e fortalecer a musculatura estabilizadora do tronco vêm sendo adotados para prevenção e recuperação desses problemas na coluna vertebral (COMERFORD e MOTTRAN, 2001; WITVROUW et al., 2004). A despeito deste panorama, pouco se sabe sobre os efeitos biomecânicos dessas práticas corporais em pessoas com lombalgia crônica inespecífica.

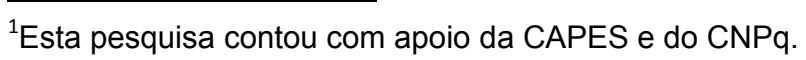


A lombalgia se manifesta principalmente, através da dor, tensão muscular e rigidez nas estruturas articulares da coluna vertebral (VAN de VEEN et al., 2005). Há evidências na literatura de que estes sintomas proporcionam mudanças no comportamento biomecânico do tronco devido às alterações no padrão de movimento e da atividade muscular das estruturas intervertebrais (WILLIANS et al., 2010). Contudo, os estudos se limitam a comparar as variáveis biomecânicas entre a população sintomática e assintomática (LAMOTH et al., 2006; VOGT et al., 2003; WONG e LEE, 2004). Este tipo de análise pode levar a conclusões limitadas já que a postura vertebral apresenta alta variabilidade inter-sujeitos (FRIGO et al., 2003; SAUNDERS et al., 2005), o que dificulta a interpretação dos resultados.

Neste sentido, torna-se interessante analisar o comportamento biomecânico que cada indivíduo apresenta com e sem dor, com o intuito de verificar alterações nos padrões de movimento corporal, além de avaliar a eficácia de diferentes abordagens terapêuticas.

A maioria dos trabalhos utiliza recursos subjetivos para o processo avaliativo como, por exemplo, o questionário de Roland Morris e a Escala Visual Analógica de Dor (HENCHOZ e KAI-LIK SO, 2008). O padrão de recrutamento dos músculos do tronco, durante a realização de determinada atividade funcional, é uma forma objetiva também empregada para avaliar os efeitos das intervenções (FERREIRA et al., 2007; TSAO e HODGES, 2008). Contudo, raríssimos são os estudos que procuram analisar a cinemática da coluna vertebral de lombálgicos antes e após um programa de exercícios físicos (CARPES et al., 2008; TAWFIK, 2001).

Portanto, o objetivo do presente estudo foi investigar se um programa de exercícios de força muscular e alongamento ativo proporcionaria alterações na função motora, na sensação subjetiva de dor, na postura estática e no padrão de movimento vertebral durante a marcha e a corrida de indivíduos com lombalgia crônica inespecífica.

\section{Materiais e métodos}

Sujeitos

Participaram deste estudo dois homens adultos (idade: 29 e 38 anos; estatura: 1,80 e 1,86 m; massa corporal: 86 e $84 \mathrm{~kg}$, respectivamente) com dor lombar crônica inespecífica. Antes de assinar o termo de consentimento livre e esclarecido, os voluntários foram orientados acerca de todos os procedimentos, riscos e benefícios associados à participação no estudo. Esta pesquisa foi aprovada pelo Comitê de Ética em Pesquisa da UNICAMP (Parecer nº 391/2007).

Programa de exercícios

Os voluntários com lombalgia foram submetidos a um programa de reabilitação que consistiu de duas sessões semanais de exercícios, com duração aproximada de uma hora, totalizando dezesseis sessões. As sessões aconteceram no centro de saúde da comunidade da universidade na área de fisioterapia e desportos. O programa de intervenção enfatizou exercícios de alongamento e de força muscular.

Para a melhora da flexibilidade optou-se pela técnica de alongamento ativo. Iniciou-se com três séries de trinta segundos, progredindo até uma única vez de noventa

Pensar a Prática, Goiânia, v. 14, n. 2, p. 1-13, maio/ago. 2011 
segundos. A intensidade foi determinada pelo voluntário, estabelecendo como limites a tensão máxima e a dor.

O programa para ganho de força muscular consistiu de três séries progressivas (ao longo das sessões) que variaram de seis a quinze repetições. Iniciou-se com uma sobrecarga baixa (exercício ativo livre), seguida de aumento gradual (exercício ativo resistido), priorizando os principais estabilizadores da pelve e do tronco.

\section{Questionário de Roland Morris e escala visual analógica de dor}

Antes e após o programa de dezesseis sessões de exercícios, os dois participantes foram avaliados através do questionário de Roland Morris e da escala subjetiva de dor, que têm como finalidade analisar a função motora e a intensidade da dor, respectivamente (NORRIS e MATTHEWS, 2008) ${ }^{2}$.

Análise biomecânica por videogrametria

Antes e após a intervenção, os participantes foram submetidos a uma avaliação biomecânica da coluna vertebral. Marcadores analérgicos, retro refletivos e planos foram fixados sobre o dorso do participante enquanto o mesmo permanecia na postura ortostática. A Figura 1 ilustra a vestimenta e a marcação do dorso.

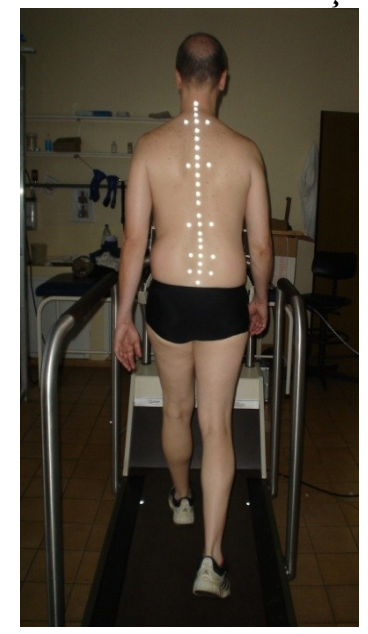

FIGURA 1 - Registro da marcação do dorso dos voluntários durante o protocolo experimental.

O teste teve início com o registro de um objeto de calibração e, em seguida, um aquecimento na esteira a uma velocidade de $1,2 \mathrm{~m} / \mathrm{s}$ durante 4 minutos para permitir uma adaptação à atividade (TAYLOR et al., 1996). Na seqüência foi feita a filmagem dos marcadores fixados sobre o dorso dos participantes, de acordo com o protocolo

\footnotetext{
${ }^{2} \mathrm{O}$ questionário de Roland Morris consiste em assinalar questões que envolvem atividades cotidianas e que o indivíduo desenvolve com limitações e/ou dor. Um maior número de respostas assinaladas indica um maior comprometimento funcional. Já a escala visual analógica de dor varia de 0 a 10 , sendo 0 ausência de dor e 10 um indicativo de dor insuportável.
}

Pensar a Prática, Goiânia, v. 14, n. 2, p. 1-13, maio/ago. 2011 
experimental que teve a seguinte ordem cronológica: registro dinâmico nas velocidades de marcha $(1.2,1.5,1.8 \mathrm{~m} / \mathrm{s})$, de corrida $(2.2,2.6,3.0 \mathrm{~m} / \mathrm{s})$ e registro postural estático.

As seqüências de imagens oriundas das três câmeras foram sincronizadas através de um sinal visual. Duas câmeras foram posicionadas posteriormente ao sujeito para registrar a movimentação da coluna, enquanto uma terceira câmera foi posicionada lateralmente, a fim de permitir a identificação dos eventos das passadas.

O método DLT (Direct Linear Transformation) permitiu reconstruir tridimensionalmente a posição dos marcadores fixados na coluna e suas coordenadas 3D foram suavizadas, em função do tempo, por meio de um filtro digital passa baixa do tipo Butterworth de quinta ordem e freqüência de corte de $6 \mathrm{~Hz}$. O modelo de representação da coluna vertebral adotado foi o mesmo proposto por Brenzikofer et al. (2000).

Neste estudo, a coluna foi analisada no plano sagital. Em cada instante do movimento, uma função polinomial foi ajustada na projeção sagital dos marcadores da coluna pelo método dos quadrados mínimos. Mediante este polinômio e de suas duas primeiras derivadas obteve-se, em cada instante medido do ciclo de locomoção, as curvaturas geométricas bidimensionais no plano sagital, em função da coordenada vertical. Posteriormente, foi quantificada a curva neutra e o componente oscilatório da coluna (CAMPOS et al., 2005; PAULA et al., 2009).

A curva neutra é a curva média de todas as curvas medidas ao longo do ciclo da passada. A neutra representa a postura inerente ao sujeito e se assemelha àquela medida na postura estática. Já o componente oscilatório mensura a movimentação da coluna em torno do componente neutro. Isto é, ao longo do ciclo, a coluna se estende e flexiona a partir da postura neutra que é tida como referência (CAMPOS et al., 2005).

\section{Variáveis investigadas}

Além de analisar a função motora e a intensidade da dor, o presente estudo mensurou as curvaturas fisiológicas da coluna na região de maior cifose torácica e lordose lombar através dos picos de curvatura geométrica tanto da curva estática (um único registro) como das dinâmicas (curvas neutras de dezesseis passadas, em cada uma das seis velocidades).

Além disto, foi avaliada também a mobilidade da coluna torácica e lombar, durante a locomoção. Para tanto, utilizaram-se as informações oriundas do componente oscilatório, na altura da região de maior cifose e lordose. A mobilidade vertebral foi avaliada de duas formas distintas.

A primeira consistiu em quantificar a amplitude de movimento através da subtração entre os picos de curvatura do componente oscilatório, obtidos ao longo dos ciclos de locomoção, nas respectivas regiões anatômicas. A segunda análise teve como meta verificar a oscilação da coluna nas regiões de maior cifose e lordose, e relacioná-las aos eventos característicos do ciclo de uma passada média, em uma velocidade de marcha $(1,5 \mathrm{~m} / \mathrm{s})$ e outra de corrida $(2,6 \mathrm{~m} / \mathrm{s})$.

Análise estatística

No presente estudo as análises estatísticas se restringiram a comparar as variáveis curvatura geométrica da cifose/lordose na situação dinâmica e amplitude de movimento da coluna torácica/lombar, antes e depois do programa de exercícios.

Para verificar se alguma das variáveis mencionadas anteriormente sofreu alterações significativas foi empregada a anova one way para testar a hipótese nula de que as médias da variável em questão não diferem entre si devido ao programa de exercícios. Nos casos em que a hipótese nula foi rejeitada, utilizou-se o teste de comparação múltipla de Tukey para constatar entre quais situações ocorreram estas distinções. Para todas as análises foi adotada a significância estatística de $5 \%(p<0,05)$.

Pensar a Prática, Goiânia, v. 14, n. 2, p. 1-13, maio/ago. 2011 


\section{Resultados}

A Tabela 1 ilustra os resultados do questionário de Roland Morris e da Escala Visual Analógica de Dor, de ambos os participantes, nas situações pré e pósintervenção.

Tabela 1 - Número de respostas assinaladas no questionário de Roland Morris e sensação subjetiva de dor (escala de 0 a 10) dos dois voluntários, antes e após o programa de exercícios.

\begin{tabular}{ccccc}
\hline \multirow{2}{*}{ Voluntários } & \multicolumn{2}{c}{$\begin{array}{c}\text { Questionário de Roland } \\
\text { Morris }\end{array}$} & Escala Visual Analógica de Dor \\
\cline { 2 - 5 } & Antes & Depois & Antes & Depois \\
\hline $\begin{array}{c}\text { Participante } \\
1\end{array}$ & 8 & 4 & 6 & 2 \\
$\begin{array}{c}\text { Participante } \\
2\end{array}$ & 8 & 1 & 5 & 1 \\
\hline
\end{tabular}

Com base na Tabela 1 nota-se que a intervenção proposta proporcionou, para ambos os sujeitos, melhora na função motora e diminuição do quadro doloroso, pois, após a intervenção houve um menor número de respostas assinaladas no questionário de Roland Morris e uma menor sensação subjetiva de dor, respectivamente.

Em decorrência do grande número de informações, as figuras 2,3 e 4 apresentam os resultados de somente um dos participantes. O comentário acerca dos resultados do outro voluntário se encontra no texto.

A Figura 2 apresenta a curvatura da região de maior cifose torácica e lordose lombar (picos de curvatura) do participante 1 em diferentes situações. Na postura estática tem-se a medida das curvaturas fisiológicas antes e depois da intervenção (boxplot da esquerda e direita, respectivamente em cada situação). De forma análoga, na postura dinâmica, têm-se as medidas das respectivas curvaturas em cada uma das dezesseis passadas consecutivas, de cada velocidade, na situação pré e pós-intervenção.

Pensar a Prática, Goiânia, v. 14, n. 2, p. 1-13, maio/ago. 2011 
Curvatura da cifose (sup.) e lordose (inf.) e m cada situaçäo

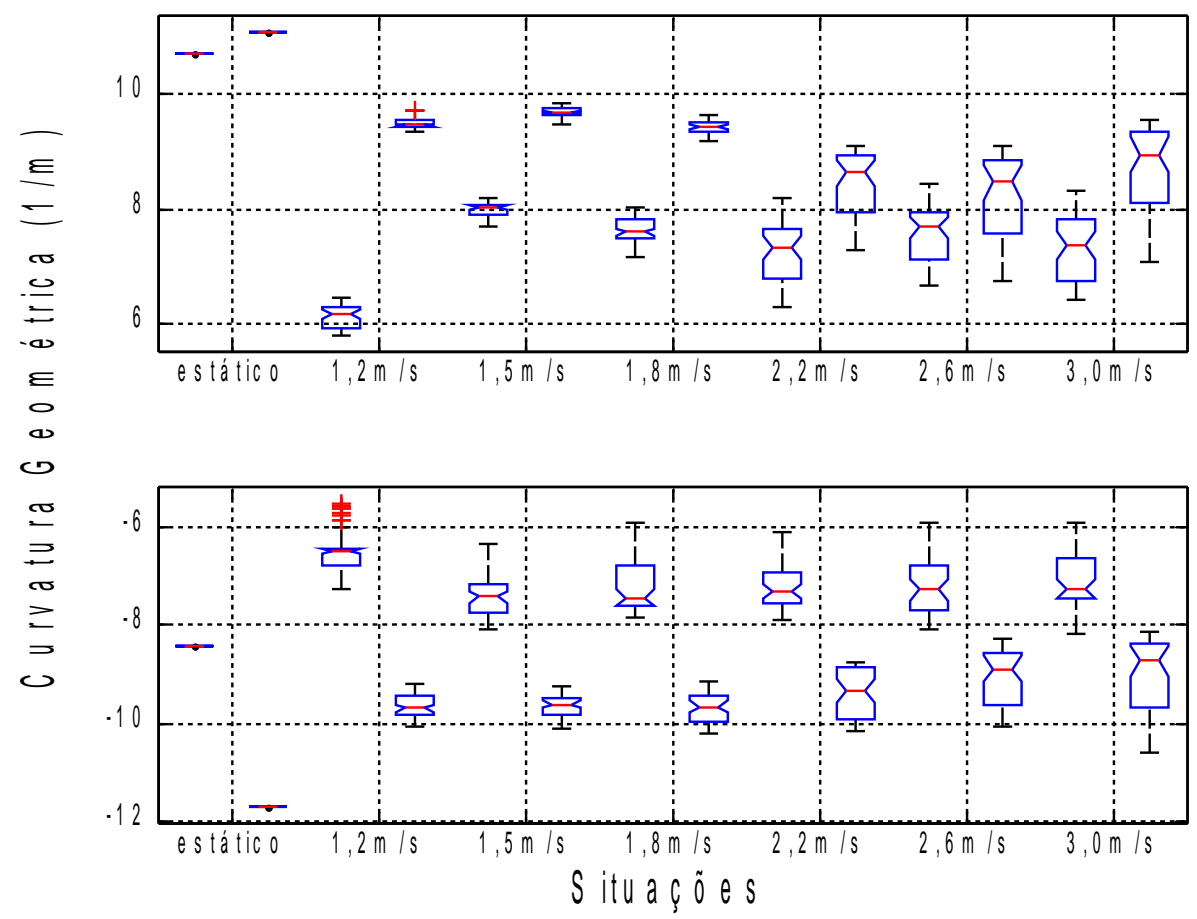

Figura 2 - Curvatura geométrica da região de maior cifose torácica (gráfico superior) e lordose lombar (gráfico inferior) do voluntário 1 nas diferentes situações, antes e após o programa de exercícios.

Com relação ao registro postural estático do participante 1, nota-se na figura 2 que a cifose torácica pouco se modificou após o programa de exercícios. Em compensação, o aumento da lordose lombar é evidente. Este comportamento também foi observado para o participante 2 .

$\mathrm{Na}$ postura dinâmica (locomoção) foram encontradas diferenças significativas $(p<0,05)$ para ambas as regiões da coluna vertebral e em todas as velocidades, sendo que depois da intervenção as curvaturas fisiológicas se tornaram mais acentuadas. Porém, o segundo voluntário apresentou este padrão somente para a região lombar já que a maior cifose torácica foi observada antes da intervenção.

Para análise da postura dinâmica, além de verificar a curvatura da região de maior cifose torácica e lordose lombar, o presente trabalho teve também como interesse quantificar a mobilidade vertebral nestas respectivas regiões da coluna, em cada uma das dezesseis passadas investigadas. A amplitude de movimento da coluna torácica e lombar foi obtida através dos valores máximos referentes aos movimentos vertebrais (flexão e extensão), em cada velocidade de locomoção e situação. A Figura 3 mostra o panorama desta comparação para o participante 1. 


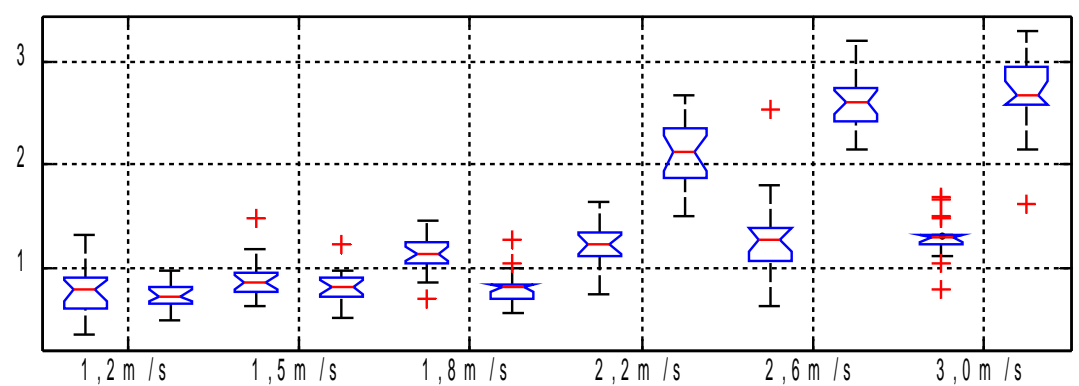

Figura 3 - Amplitude de movimento da coluna torácica (gráfico superior) e lombar (gráfico inferior) do voluntário 1 , em cada situação, antes e após o programa de exercícios.

Vale descrever que, em cada velocidade, temos dois boxplots sendo que o da esquerda representa a condição pré e o da direita pós participação no programa de exercícios. $\mathrm{O}$ voluntário 1 apresentou valores significativamente maiores de amplitude de movimento da coluna torácica, somente durante a corrida, na situação pósintervenção. Por outro lado, a região lombar teve diminuições significativas na amplitude de movimento após o programa de exercícios. O voluntário 2 apresentou comportamento vertebral muito semelhante ao do primeiro avaliado, exceto para a coluna torácica, que se mostrou sensível à intervenção com o aumento na amplitude para todas as velocidades.

Outra análise utilizada foi verificar a oscilação da postura da coluna vertebral da região de maior cifose torácica e lordose lombar, ao longo do ciclo de locomoção. Para esta abordagem foram selecionados os dados referentes a uma única velocidade de marcha e de corrida, com suas respectivas passadas médias (Figura 4). Este artifício teve como finalidade reduzir o número de informações para facilitar a visualização dos dados. Vale destacar que esta seleção não diminuiu a qualidade das informações, pois, as velocidades medianas $(1,5$ e $2,6 \mathrm{~m} / \mathrm{s})$ representam com propriedade o grupo marcha $\mathrm{e}$ corrida. 

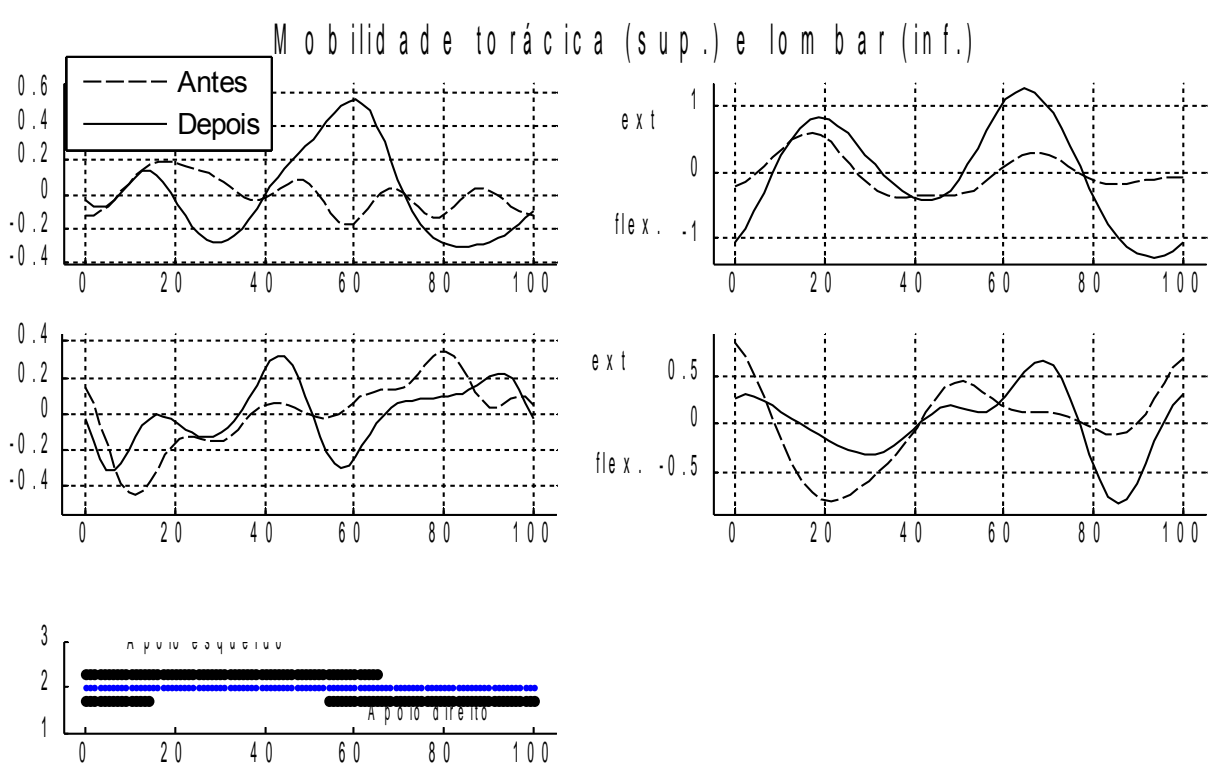

Figura 4 - Oscilação da mobilidade torácica (gráficos superiores) e lombar (gráficos inferiores), ao longo do ciclo de locomoção, na marcha (gráficos da esquerda) e corrida (gráficos da direita) antes e após a intervenção com o programa de exercícios. Dados do voluntário 2.

Na Figura 4 temos a oscilação da postura vertebral da região de maior cifose torácica e lordose lombar, ao longo do ciclo de locomoção do voluntário 2. Nos gráficos desta figura, os valores positivos e negativos do componente oscilatório representam, respectivamente, flexão e extensão da coluna vertebral. Dessa forma, quando houve incremento da curvatura na região torácica, correspondeu a um aumento da cifose. Já na lombar, ocorreu um aumento da lordose quando a curvatura diminuiu. Quando a curvatura geométrica do componente oscilatório foi nula significou que naquele instante do ciclo a coluna torácica e/ou lombar se encontrava na postura neutra.

Com base na Figura 4 nota-se que durante a marcha (gráficos da esquerda), tanto a coluna torácica como a lombar apresentaram diferenças antes e após o programa de exercícios, principalmente, durante o segundo duplo apoio (DA). Neste período do ciclo houve uma inversão no movimento vertebral já que a torácica passou a se flexionar e a lombar a se estender após a intervenção. Na corrida (gráficos da direita), verifica-se que as principais modificações na movimentação da coluna torácica e lombar estão relacionadas à magnitude da oscilação, conforme já foi discutido anteriormente (amplitude).

Já o voluntário 1, durante a marcha, apresentou uma inversão de movimento na coluna torácica e lombar durante o apoio simples esquerdo, pois, depois da intervenção, ambas as regiões, ao invés de se estenderem, passaram a se flexionar neste período do ciclo. Na corrida, a torácica se distinguiu principalmente em relação à magnitude da oscilação. Já a lombar apresentou uma inversão no movimento vertebral durante a primeira fase de vôo, pois, ao contrário do que ocorreu na avaliação prévia, a coluna passou a se flexionar no instante em que o avaliado perde o contato com o solo.

É importante destacar que os dois voluntários, independentemente da forma de locomoção e da região da coluna vertebral, apresentaram uma mudança na seqüência temporal de ocorrência dos picos devido à intervenção.

\section{Discussão}

Pensar a Prática, Goiânia, v. 14, n. 2, p. 1-13, maio/ago. 2011 
O presente trabalho visou verificar se o programa de exercícios empregado proporciona modificações estruturais e funcionais na coluna vertebral de indivíduos com lombalgia crônica inespecífica. Para tanto, foi aplicado um questionário e feita uma análise cinemática da coluna durante a postura estática e dinâmica.

Com base no resultado da escala subjetiva de dor e do questionário de Roland Morris nota-se que os dois voluntários tiveram diminuição do quadro doloroso e melhora na função motora, respectivamente. Na literatura, a classificação efetiva das síndromes lombares são consideradas a partir de três unidades na escala visual analógica, pois, valores abaixo deste limiar são caracterizados como dores de baixa intensidade (VOGT et al., 2003).

Neste sentido, observa-se que os participantes deste estudo relataram algias de nível moderado na situação prévia e de baixa intensidade após a intervenção com o programa de exercícios, o que denota a importância da mesma no alívio da dor. Apesar de, aparentemente, o segundo voluntário ter obtido avanços mais promissores em ambos os quesitos, é importante destacar que não existe uma forma única ou melhor para reabilitar o indivíduo acometido pela lombalgia, pois, diferentes sujeitos podem responder de diferentes formas a um mesmo tipo de intervenção (MAY e DONELSON, 2008).

Além da diminuição do quadro álgico e melhora funcional, constatada pela percepção subjetiva do avaliado, verificou-se também modificações estruturais e funcionais na coluna vertebral em decorrência do programa de intervenção. $\mathrm{O}$ aumento da cifose torácica e da lordose lombar demonstra que houveram mudanças estruturais na postura dos participantes. Jackson et al. (2000) destacam que o alinhamento da coluna, no plano sagital, é uma característica única e, em tese, não deve sofrer alterações significativas ao longo de alguns anos, a não ser que o indivíduo seja submetido a algum tipo de intervenção.

Acredita-se que o aumento nas curvaturas fisiológicas da coluna torácica e lombar esteja relacionado às alterações no comportamento motor dos músculos do tronco, pois, o sistema neuromotor está intimamente atrelado à postura vertebral (PANJABI, 2003). Neste sentido, especula-se que estes resultados corroboram com as afirmações de Panjabi (2003) de que indivíduos acometidos por dor lombar apresentam uma diminuição da zona neutra devido à maior resistência das estruturas intervertebrais. Este comportamento parece ser uma tentativa do sistema neuromuscular em preservar a integridade física das estruturas músculo-esqueléticas e/ou para evitar a dor (VOGT et al., 2003; WILLIANS et al., 2010).

Outra variável quantitativa avaliada foi à mobilidade vertebral nas regiões de maior cifose torácica e lordose lombar. A diminuição na amplitude de movimento da coluna lombar após a intervenção corrobora com os achados de Carpes et al. (2008), apesar das diferenças metodológicas em relação ao modelo biomecânico empregado para representar a coluna vertebral e aos exercícios utilizados. Por outro lado, o aumento na amplitude de movimento na região torácica pode estar relacionado a uma necessidade de oscilação da estrutura vertebral em decorrência da demanda mecânica já que a região lombar se encontra menos móvel após a intervenção.

Panjabi (2003) relata que indivíduos acometidos por dor lombar podem apresentar tanto diminuição quanto aumento na amplitude de movimento vertebral. Esta variabilidade está relacionada à ação de diferentes músculos e estratégias de controle que estão envolvidas no mecanismo de estabilidade intervertebral. Neste sentido, podese dizer que o sistema neuromotor opera de forma redundante já que muitos músculos desempenham funções similares (HODGES, 2003).

Pensar a Prática, Goiânia, v. 14, n. 2, p. 1-13, maio/ago. 2011 
10.5216/rpp.v14i2.14603

Outro fato interessante é que o programa de exercícios proporcionou alterações nos instantes de ocorrência dos picos de curvatura geométrica durante o ciclo de locomoção, de forma que a coluna vertebral passou a se flexionar e/ou a se estender, até o seu máximo, num evento distinto daquele observado na situação prévia. Este fenômeno pode estar relacionado às alterações no padrão de recrutamento motor dos músculos afetados pela síndrome dolorosa, que costumam proporcionar ativações inadequadas na freqüência e no desenvolvimento de força (NEWMAN et al., 1996; VOGT et al., 2003).

Apesar de ser difícil estabelecer qual padrão de movimento é o mais adequado, especula-se que aquele visualizado após a intervenção seja o mais apropriado já que nestas circunstâncias os voluntários tiveram uma redução no quadro álgico e melhora na função motora.

\section{Referências}

ANDERSSON, G.B. Epidemiological features of chronic low back pain. The Lancet, v.354, n.9178, p.581-585, 1999. Disponível em: http://dx.doi.org/10.1016/S01406736(99)01312-4. Acesso em: 19 mai. 2010.

BRENZIKOFER, R.; BARROS, R.M.L.; LIMA FILHO, E.C.; TOMA, E.; BORDINI, L.S. Alterações no dorso e coluna vertebral durante a marcha. Revista Brasileira de Biomecânica, v.1, n.1, p.21-6, 2000.

CAMPOS, M.H.; BRENZIKOFER, R.; DEPRÁ, P.P.; PEGORETTI, C.; WITTIG, D.S.; BENETTI, F.A. Metodologia para quantificação da curva neutra da coluna vertebral durante a marcha: Estudo piloto. In: XI CONGRESSO BRASILEIRO DE BIOMECÂNICA, v.1, 2005, João Pessoa. Anais do XI Congresso Brasileiro de Biomecânica. João Pessoa: 2005, p. 131-5.

CARPES, F.P.; REINEHR, F.B.; MOTA, C.B. Effects of a program for trunk strength and stability on pain, low back and pelvis kinematics, and body balance: A pilot study. Journal of Bodywork and Movement Therapies, v.12, p.22-30, 2008. Disponível em: http://dx.doi.org/10.1016/j.jbmt.2007.05.001. Acesso em: 19 mai. 2010.

COMERFORD, M. J.; MOTTRAN, S. L. Functional stability re-training: principles and strategies for managing mechanical dysfunction. Manual Therapy. v.6(1). 2001. Disponível em: doi:10.1054/math.2000.0389. Acesso em: 07 jul. 2009.

FERREIRA, M.L.; FERREIRA, P.H.; HODGES, P.W. Changes in postural activity of the trunk muscles following spinal manipulative therapy. Manual Therapy, v.12, p.240248, 2007. Disponível em: http://dx.doi.org/10.1016/j.math.2006.06.015. Acesso em: 19 mai. 2010.

FRIGO, C.; CARABALONA, R.; MURA, M.D.; NEGRINI, S. The upper body segmental movements during walking by young females. Clinical Biomechanics, v.18, p.419-25, 2003. Disponível em: http://dx.doi.org/10.1016/S0268-0033(03)00028-7. Acesso em: 19 mai. 2010.

Pensar a Prática, Goiânia, v. 14, n. 2, p. 1-13, maio/ago. 2011 
10.5216/rpp.v14i2.14603

HENCHOZ, Y.; KAI-LIK SO, A. Exercise and nonspecific low back pain: A literature review. Joint Bone Spine, v.75, p.533-539, 2008. Disponível em: http://dx.doi.org/10.1016/j.jbspin.2008.03.003. Acesso em: 19 mai. 2010.

HODGES, P.W. Core stability exercise in chronic low back pain. Orthopedic Clinics of North America, v.34, p.245-254, 2003. Disponível em: http://dx.doi.org/10.1016/S0030-5898(03)00003-8. Acesso em: 19 mai. 2010.

JACKSON, R. P. Lumbopelvic lordosis and pelvic balance on repeated standing lateral radiographs of adult volunteers and untreated patients with constant low back pain. Spine, v. 25, n. 5, p. 575-86, 2000. Disponível em: http://www.ncbi.nlm.nih.gov/pubmed/10749634. Acesso em: 19 mai. 2010.

LAMOTH, C. J. C.; DAFFERTSHOFER, A.; MEIJER, O.G.; BEEK, P.J. How do persons with chronic low back pain speed up and slow down? Trunk-pelvis coordination and lumbar erector spine activity during gait. Gait \& Posture, v.23, p.230-239, 2006. Disponível em: http://dx.doi.org/10.1016/j.gaitpost.2005.02.006. Acesso em: 19 mai. 2010.

MAY, S.; DONELSON, R. Evidence-informed management of chronic low back pain with the McKenzie method. The Spine Journal, v. 8, p. 134-41, 2008. Disponível em: http://dx.doi.org/10.1016/j.spinee.2007.10.017. Acesso em: 19 mai. 2010.

NEWMAN, N. GRACOVETSKY, S.; ITOI, M.; ZUCHERMAN, J.; RICHARDS, M.; DURAND, P.; XELLER, C.; CARR, D. Can the computerized physical examination differentiate normal subjects from abnormal subjects with benign mechanical low back pain? Clinical Biomechanics, v. 11, n. 8, p. 466-73, 1996. Disponível em: http://dx.doi.org/10.1016/S0268-0033(96)00022-8. Acesso em: 19 mai. 2010.

NORRIS, C.; MATTHEWS, M. The role of an integrated back stability program in patients with chronic low back pain. Complementary Therapies in Clinical Practice, v.14, p.255-263, 2008. Disponível em: http://dx.doi.org/10.1016/j.ctcp.2008.06.001. Acesso em: 19 mai. 2010.

PANJABI, M. M. Clinical spinal stability and low back pain. Journal of Electromyography and Kinesiology, v. 13, p. 371-379, 2003. Disponível em: http://dx.doi.org/10.1016/S1050-6411(03)00044-0. Acesso em: 19 mai. 2010.

PAULA, M.C.; CAMPOS, M.H.; MEIRELLES, A.A.; MORAES, M.A.A.; BRENZIKOFER, R. Análise da amplitude de movimentação da coluna vertebral, freqüência e comprimento da passada durante a marcha e a corrida. Revista Brasileira de Biomecânica, v.10, p. 1-8, 2009. Disponível em: http://citrus.uspnet.usp.br/biomecan/ojs/index.php/rbb/article/view/107. Acesso em: 19 mai. 2010.

SAUNDERS, S. W.; SCHACHE, A. RATH, D.; HODGES, P.W. Changes in three dimensional lumbo-pelvic kinematics and trunk muscle activity with speed and mode locomotion. Clinical Biomechanics, v.20, p. 784-93, 2005. Disponível em: http://dx.doi.org/10.1016/j.clinbiomech.2005.04.004. Acesso em: 19 mai. 2010.

Pensar a Prática, Goiânia, v. 14, n. 2, p. 1-13, maio/ago. 2011 
TAYLOR, N. F.; EVANS, O. M.; GOLDIE, P. A. Angular movements of the lumbar spine and pélvis can be reliably measured after 4 minutes of treadmill walking. Clinical Biomechanics, v.11, p. 484-86, 1996. Disponível em: http://dx.doi.org/10.1016/S02680033(96)00036-8. Acesso em: 19 mai. 2010.

TAWFIK, B. Symmetry and linearity of trunk function in subjects with non-specific low back pain. Clinical Biomechanics, v.16, p.114-120, 2001. Disponível em: http://dx.doi.org/10.1016/S0268-0033(00)00079-6. Acesso em: 19 mai. 2010.

TSAO, H.; HODGES, P.W. Persistence of improvements in postural strategies following motor control training in people with recurrent low back pain. Journal of Electromyography and Kinesiology, v. 18, p. 559-567, 2008. Disponível em: http://dx.doi.org/10.1016/j.jelekin.2006.10.012. Acesso em: 19 mai. 2010.

VAN DE VEEN, E.A.; DE VET, H.C.; POOL, J.J.; SCHULLER, W.; DE ZOETE, A.; BOUTER, L.M. Variance in manual treatment of nonspecific low back pain between orthomanual physicians, manual therapists, and chiropractors. Journal of Manipulative and Physiological Therapeutics, v. 28, n.2, p. 108-116, 2005. Disponível em: http://dx.doi.org/10.1016/j.jmpt.2005.01.008 . Acesso em: 19 mai. 2010.

VOGT, L.; PFEIFER, K.; BANZER, W. Neuromuscular control of walking with chronic low back pain. Manual Therapy, v. 8, n. 1, p. 21-28. 2003. Disponível em: http://dx.doi.org/10.1054/math.2002.0476. Acesso em: 19 mai. 2010.

WILLIAMS, J.M.; HAQ, I.; LEE, R.Y. Is pain the cause of altered biomechanical functions in back sufferers? Human Movement Science, v. 29, p. 311-325, 2010. Disponível em: http://dx.doi.org/10.1016/j.humov.2009.12.001. Acesso em: 19 mai. 2010.

WITVROUW, E.; MAHIEU, N.; DANNEELS, L.; McNAIR, P. Stretching and Injury Prevention: An Obscure Relationship. Sports Medicine. v.34(7). p. 443-9. 2004.

WONG, T.K.T; LEE, R.Y.W. Effects of low back pain on the relationship between the movements of the lumbar spine and hip. Human Movement Science, v. 23, p. 21-34, 2004. Disponível em: http://dx.doi.org/10.1016/j.humov.2004.03.004. Acesso em: 19 mai. 2010.

\title{
EFFECTS OF AN EXERCISE TRAINING PROGRAM ON THE VERTEBRAL POSTURE OF LOW BACK PAIN SUBJECTS
}

\begin{abstract}
The aim of this work was to evaluate the effects of an exercise training program in the vertebral posture of two individuals with unspecific chronic low back pain (29 and 38 years; 1.80 and $1.86 \mathrm{~m} ; 86$ and $84 \mathrm{~kg}$ ). The 2D geometric curvature of the vertebral column, in the sagittal plane, was quantified by videogrametry in the standing posture, walking $(1.2,1.5$ and $1.8 \mathrm{~m} / \mathrm{s})$ and running $(2.2,2.6$ and $3.0 \mathrm{~m} / \mathrm{s})$. The spine movement pattern changed after the intervention. The range of motion of the lumbar spine decreased. Furthermore, the lumbar lordosis and the thoracic kyphosis
\end{abstract}


10.5216/rpp.v14i2.14603

were increased. Associated to these, the pain decreased and it was an improvement of the motor function, suggesting a better movement pattern.

Keywords: Gait. Biomechanics. Spine. Low Back Pain.

\section{EFECTOS DE UN PROGRAMA DE EJERCICIOS EN LA POSTURA DE LA COLUMNA DE PERSONAS CON DOLOR LUMBAR.}

Resumen: El objetivo de este estudio fue examinar los efectos de un programa de ejercicios en la postura de la columna de dos personas con dolor crónico lumbar inespecífico ( 29 y 38 años, 1,80 y 1,86 m, $86 \mathrm{~kg}$ y 84). La curvatura geométrica 2D de la columna vertebral, en el plano sagital, se cuantificó con videogrametría en la postura estatica, en la marcha $(1.2,1.5$ y $1.8 \mathrm{~m} / \mathrm{s})$ y en la raza $(2.2,2.6$ y $3.0 \mathrm{~m} / \mathrm{s})$. El patrón de movimiento de la columna ha cambiado después de el programa de ejercicios. Hubo una disminución de la amplitud de movimiento en la región lumbar. Hubo un aumento de la lordosis lumbar y de la cifosis dorsal. Además de estos cambios biomecánicos, hubo una disminución del dolor y una mejoría de la función motora, lo que sugiere un movimiento más adecuado.

Palabras claves: Marcha. Biomecánica. Columna. Dolor de Espalda.

Endereço para correspondência:

mariohcampos@gmail.com

Mário Hebling Campos

Universidade Federal de Goiás

Faculdade de Educação Física

Campus Samambaia, Caixa Postal 131

CEP: 74001-970 Goiânia - Goiás - Brasil

Pensar a Prática, Goiânia, v. 14, n. 2, p. 1-13, maio/ago. 2011 